\title{
Acolhimento em saúde mental na atenção primária à saúde no contexto da pandemia da COVID-19
}

\author{
Welcome in mental health in primary health care in the context of the COVID-19 pandemic \\ Bienvenidos a la salud mental en la atención primaria de salud en el contexto de la pandemia
}

COVID-19

Recebido: 05/06/2021 | Revisado: 13/06/2021 | Aceito: 16/06/2021 | Publicado: 30/06/2021

Thamires Pereira de Figueiredo ORCID: https://orcid.org/0000-0002-5095-4510 Centro Universitário de Patos, Brasil E-mail: thamiresrck20@gmail.com

Milena Nunes Alves de Sousa

ORCID: https://orcid.org/0000-0003-1684-7655 Centro Universitário de Patos, Brasil E-mail: milenanunes@fiponline.edu.br

Hirisleide Bezerra Alves

ORCID: https://orcid.org/0000-0002-5779-1291 Centro Universitário de Patos, Brasil

E-mail: hirisleidealves@ fiponline.edu.br

\begin{abstract}
Resumo
Objetivo. Analisar o acolhimento em saúde mental na atenção primária à saúde no contexto da pandemia da COVID19. Metodologia. Foram utilizados artigos na íntegra, disponíveis nas bases dados Medical Publications (PubMed) e Literatura Latino-americana e do Caribe em Ciências da Saúde (LILACS). Para a busca, utilizaram-se as palavraschave: "Mental Health Assistance", "Mental Health", "Family Health Strategy", "Unified Health System", "Health Personnel", "User Embracement" e "COVID-19"; obtidos de acordo com os Descritores de Ciências da Saúde (DeCS), tendo como operadores booleano "E/AND" e "OU/OR". Posteriormente à aplicação dos critérios de inclusão definidos, 15 artigos foram selecionados para revisão. Resultados. Todos os artigos selecionados para esta revisão abordaram estratégias de atuação da Estratégia Saúde da Família no contexto da atenção à saúde mental, tanto antes quanto no período da pandemia. Além disso, muitos estudos trouxeram limitações como dificuldades de acesso, baixa resolutividade no âmbito da Atenção Básica, ausência de qualificação dos profissionais, além de falta de acesso à internet por parte dos usuários e baixa adesão às novas abordagens no contexto da pandemia. Conclusão. Há uma implementação de novas práticas que se adaptem ao novo cenário como, por exemplo, o teleatendimento, por meio de videochamadas e ligações. Apesar disso, essas práticas possuem limitações em decorrência do perfil dos usuários. Dessa forma, são necessárias novas estratégias para acolhimento que possam incluir todos os usuários do serviço.
\end{abstract}

Palavras-chave: Assistência à saúde mental; Estratégia saúde da família; Infecção por coronavirus.

\begin{abstract}
Objective. Analyze, in the scientific production, reception in mental health in primary health care in the context of the COVID-19 pandemic. Methodology. Full articles from the Medical Publications (PubMed) and Latin American and Caribbean Health Sciences (LILACS) databases were used. For the search, the following keywords were used: "Mental Health Assistance", "Mental Health", "Family Health Strategy", "Unified Health System", "Health Personnel", "User Embracement" and "COVID-19"; obtained according to the Health Sciences Descriptors (DeCS), having as Boolean operators "AND/AND" and "OR/OR". After applying the inclusion criteria defined, 15 articles were selected for review. Results. All the articles selected for this review addressed the Family Health Strategy's action strategies in the context of mental health care, both before and during the pandemic period. In addition, many studies also brought limitations such as difficult access, low resolution in primary care, lack of professional qualifications, in addition to lack of internet access by users and low adherence to new approaches in the context of the pandemic. Conclusion. It can be concluded that there is an implementation of new practices that adapt to the new scenario, such as teleservice, through video calls and calls. Despite this, these practices have limitations due to the profile of the users. Thus, new welcoming strategies are needed that can include all service users.
\end{abstract}

Keywords: Mental health care; Family health strategy; Infection from coronavirus. 


\begin{abstract}
Resumen
Objetivo. Analizar, en la producción científica, la acogida en salud mental en la atención primaria de salud en el contexto de la pandemia COVID-19. Metodología. Se utilizaron artículos completos de las bases de datos de Publicaciones Médicas (PubMed) y Ciencias de la Salud de América Latina y el Caribe (LILACS). Para la búsqueda se utilizaron las siguientes palabras clave: "Asistencia en Salud Mental", "Salud Mental", "Estrategia de Salud de la Familia", "Sistema Único de Salud", "Personal de Salud", "Aceptación del Usuario" y "COVID-19"; obtenido según los Descriptores de Ciencias de la Salud (DeCS), teniendo como operadores booleanos "Y / Y" y "O / O". Después de aplicar los criterios de inclusión definidos, se seleccionaron 15 artículos para su revisión. Resultados. Todos los artículos seleccionados para esta revisión abordaron las estrategias de acción de la Estrategia de Salud de la Familia en el contexto de la atención de la salud mental, tanto antes como durante el período pandémico. Además, muchos estudios también trajeron limitaciones como la dificultad de acceso, la baja resolución en atención primaria, la falta de cualificación profesional, además de la falta de acceso a internet por parte de los usuarios y la baja adherencia a nuevos enfoques en el contexto de la pandemia. Conclusión. Se puede concluir que existe una implementación de nuevas prácticas que se adaptan al nuevo escenario, como el teleservicio, a través de videollamadas y llamadas. A pesar de esto, estas prácticas tienen limitaciones debido al perfil de los usuarios. Por tanto, se necesitan nuevas estrategias de acogida que puedan incluir a todos los usuarios del servicio.
\end{abstract}

Palabras clave: Atención a la salud mental; Estrategia de salud familiar; Infección por coronavirus.

\title{
1. Introdução
}

O Programa de Saúde da Família (PSF), criado no início da década 1990, foi idealizado como uma estratégia de reorientação do modelo assistencial, visando superar a fragmentação presente no modelo biomédico, a partir do estabelecimento de vínculos positivos entre a equipe de saúde e os cidadãos e grupos familiares moradores em território de adscrição (Ribeiro, Caccia-Bava \& Guanes-Lorenzi, 2013).

Criado em 1994 pelo Ministério da Saúde, visando modificar a prática da Atenção Básica (AB), passou a ser denominado de Estratégia de Saúde da Família (ESF) deixando de ser apenas um programa para se tornar a estratégia principal de organização da AB (Gazinato \& Castro, 2014b). Entre seus objetivos básicos, a ESF assume a assistência humanizada e a criação de vínculos que possibilitam aos profissionais intervir na comunidade por meio de ações específicas que produzem conhecimento sobre a prevenção e promoção da saúde (Alves \& Aerts, 2011).

No quadro das diretrizes nacionais de saúde, a Saúde da Família é descrita como estratégia para organização de todo o sistema de Atenção Primária à Saúde (APS), que é, por sua vez, vista como porta de entrada do Sistema Único de Saúde (SUS) e como contato preferencial dos cidadãos com esse Sistema (Brasil, 2006). Assim, o cuidado à Saúde Mental é considerado como uma das responsabilidades da ESF, já que muitas situações dessa natureza são experimentadas pelas famílias atendidas por essas equipes (Ribeiro, Caccia-Bava \& Guanes-Lorenzi, 2013).

A Reforma Sanitária, com sua ênfase na APS, na defesa do controle social, da universalidade e da integralidade, apresenta compatibilidades evidentes com a Reforma Psiquiátrica, levando à necessidade de reflexão sobre práticas de saúde mental na APS. A Reforma Psiquiátrica brasileira é descrita como processo político-cultural, que envolve diversos atores sociais na transformação de saberes e práticas dirigidos aos transtornos mentais (Souza, 2012).

Nos últimos anos, o Ministério da Saúde, mediante políticas de expansão, formulação, formação e avaliação da AB, vem estimulando ações que remetem a dimensão subjetiva dos usuários e aos problemas mais graves de saúde mental da população neste nível de atenção. A ESF, tomada enquanto diretriz para reorganização da AB no contexto do SUS, tornou-se fundamental para a atenção das pessoas portadoras de transtornos mentais e seus familiares; com base no trabalho organizado e mediante ações comunitárias que favorecem a inclusão social destas no território onde vivem e trabalham (Correia, Barros \& Colvero, 2011).

A equipe de saúde da família deve desenvolver propostas de cuidados que incluam a Saúde Mental, partindo das situações observadas em seu cotidiano profissional e do conhecimento dos recursos disponíveis (Souza, 2004). Tem sido 
crescente na ESF a demanda pela atenção aos transtornos psíquicos leves mais prevalentes, manifestados sob diferentes formas, entre elas os quadros depressivos e os transtornos de ansiedade (Scóz \& Fenili, 2003).

Importante frisar que, a saúde mental e sua abordagem na Atenção Primária à Saúde (APS) ainda configuram-se em uma temática bem controversa como, por exemplo, qual o papel da APS no cuidado ao paciente em saúde mental (acolhimento, atendimento ou rastreamento), quais as potencialidades e limitações no cuidado e os riscos de experienciar processos concebidos pelas autoras como "empurroterapia", "cronicidade" e "supermedicalização" dos transtornos mentais, pontos evidenciados mediante a pandemia causada pelo novo coronavírus (SARS-COV-2) (Moraes, Araújo, Porto, Trajano \& Sousa, 2021).

Desde o primeiro surto da pandemia da doença coronavírus 2019 (COVID-19) no final de dezembro de 2019 (Arruda, Martins, Silva \& Sousa, 2020; Bezerra et al., 20210; Baloch, Baloch, Zheng \& Pei, 2020; Sousa, Estrela \& Bezerra, 2020) 82.933 casos foram confirmados e 4.633 pessoas morreram na China continental até 14 de maio de 2020 (Nabuco, Oliveira \& Afonso, 2020). No Brasil, o primeiro caso da doença foi notificado em 25 de fevereiro de 2020 (BRASIL, 2020), e o número de pessoas afetadas pelo vírus vem crescendo gradativamente desde então. Nesse sentido, considerando-se a falta de recursos ao enfrentamento da pandemia, causada por um agente desconhecido até então, a principal medida de prevenção da disseminação da COVID-19 consistiu no distanciamento social, exigindo isolamento domiciliar das pessoas (Duarte, Santos, Lima, Giordani \& Trentini, 2020).

Em uma revisão do tipo Umbrella descobriu-se que a depressão, transtornos de ansiedade, transtornos do humor, sintomas de estresse pós-traumático, transtornos do sono, pânico, estigmatização, baixa autoestima, falta de autocontrole são altamente prevalentes entre indivíduos impactados com isolamento físico (Hossain, Sultana \& Purohit, 2020). Além do isolamento social, fatores como desemprego, óbitos e incertezas quanto à vacina contribuem ao aumento da incidência de transtornos mentais na população neste período.

No contexto da pandemia da COVID-19, a APS apresenta papel fundamental tanto na assistência inicial aos casos suspeitos, como também na área de saúde mental. Indivíduos que perderam familiares, estão sob isolamento, desempregados, sem renda, em condições de vulnerabilidade, enfrentando violência doméstica, caracterizam grupos com prevalência de transtornos mentais. Diante disso, ressalta-se a necessidade de avaliação das estratégias adotadas pelas equipes da APS no contexto de saúde mental, durante a pandemia, visando uma assistência adequada e segura aos pacientes, contemplando integralmente suas demandas. Sendo assim, o objetivo do presente estudo foi analisar, com base em estudos publicados em periódicos, o acolhimento em saúde mental na atenção primária à saúde no contexto da pandemia da COVID-19.

\section{Metodologia}

O presente trabalho consistiu em uma pesquisa qualitativa de caráter descritivo, caracterizada como Revisão Integrativa da Literatura (RIL). Para a realização deste estudo foram observadas seis diretrizes pré-estabelecidas em um protocolo de pesquisa: 1) escolha do tema e a questão norteadora; 2) estabelecimento dos critérios para inclusão e exclusão de estudos para seleção da amostra; 3) definição das informações a serem extraídas da amostra; 4) avaliação/análise crítica dos estudos incluídos na amostra; 5) discussão e interpretação dos resultados e 6) apresentação da revisão de forma objetiva e clara (Mowbray, Wilkinson, \& Tse, 2014).

Na primeira etapa o tema foi delimitado a partir da seguinte questão norteadora: "Quais as estratégias de atuação da equipe de saúde da família na atenção à saúde mental no contexto da pandemia da COVID-19?”. Durante a etapa subsequente foram estabelecidos os seguintes critérios de inclusão: foram utilizados apenas estudos observacionais que envolviam a realização de ações de acolhimento em saúde mental no âmbito da estratégia da saúde da família, sem restrição de data, nos idiomas inglês e português. Os critérios de exclusão foram: estudos não relacionados ao tema, estudos em espanhol, artigos de 
revisão, cartas ao editor/editoriais, opiniões pessoais, livros/capítulos de livros, livros didáticos, relatórios, resumos de conferências e patentes. Foram utilizadas as bases de dados Literatura Latino-americana e do Caribe em Ciências da Saúde (LILACS) e Medical Publications (PubMed), como fontes primárias de estudo. Uma busca manual também foi realizada por meio de uma análise sistematizada das referências dos artigos elegíveis.

Os Descritores em Ciências da Saúde (DECS) foram utilizados para selecionar os descritores de busca com os operadores booleanos "AND" e "OR". A estratégia de busca incluiu as seguintes palavras-chave: "Mental Heatlh Assistance", "Mental Health", "Family Health Strategy", "Unified Health System", "Health Personnel", "User Embracement" e "COVID19". Os resultados obtidos foram exportados para o software EndNote Web ${ }^{\mathrm{TM}}$ (Thomson Reuters, Toronto, Canadá) e as duplicatas foram removidas. Os resultados restantes foram exportados para o Microsoft Word ${ }^{T M} 2013$ (Microsofttm Ltd, Whashington, EUA) e as duplicatas restantes foram removidas manualmente.

A busca foi realizada em 14 de abril de 2021, sendo localizados um total de 50.844 artigos, sendo 3.948 na base de dados LILACS e 46.896 na PubMed (Tabela 1).

Tabela 1. Combinação de descritores para levantamento de estudos para esta revisão integrativa.

\begin{tabular}{lccc}
\hline \multirow{2}{*}{ Descritores } & \multicolumn{3}{c}{ Bases de Dados } \\
\cline { 2 - 4 } & LILACS & PubMed & Total \\
\hline mental health assistance OR mental health AND family health strategy & 653 & 4486 & 5139 \\
mental health assistance OR mental health AND health personnel & 2377 & 34804 & 37181 \\
mental health assistance OR mental health AND user embracement & 120 & 24 & 144 \\
mental health assistance OR mental health AND unified health system & 510 & 319 & 829 \\
mental health assistance OR mental health AND COVID-19 & 288 & 7263 & 7551 \\
Número total de artigos & $\mathbf{3 9 4 8}$ & $\mathbf{4 6 8 9 6}$ & $\mathbf{5 0 8 4 4}$ \\
\hline
\end{tabular}

Fonte: Dados da Pesquisa (2021).

Após a identificação nas bases de dados, foram eliminados 37.565 artigos duplicados. Além disso, posterior a aplicação dos filtros, foram excluídos mais 13.319 artigos. Em seguida, 20 trabalhos passaram por uma análise criteriosa do título e resumo, excluindo-se cinco artigos não relacionados aos objetivos da presente pesquisa, mantendo-se, assim, para leitura do texto na íntegra, 15 artigos, os quais foram selecionados para esta revisão (Figura 1). 
Figura 1. Fluxograma do processo de seleção dos artigos incluídos no presente estudo.

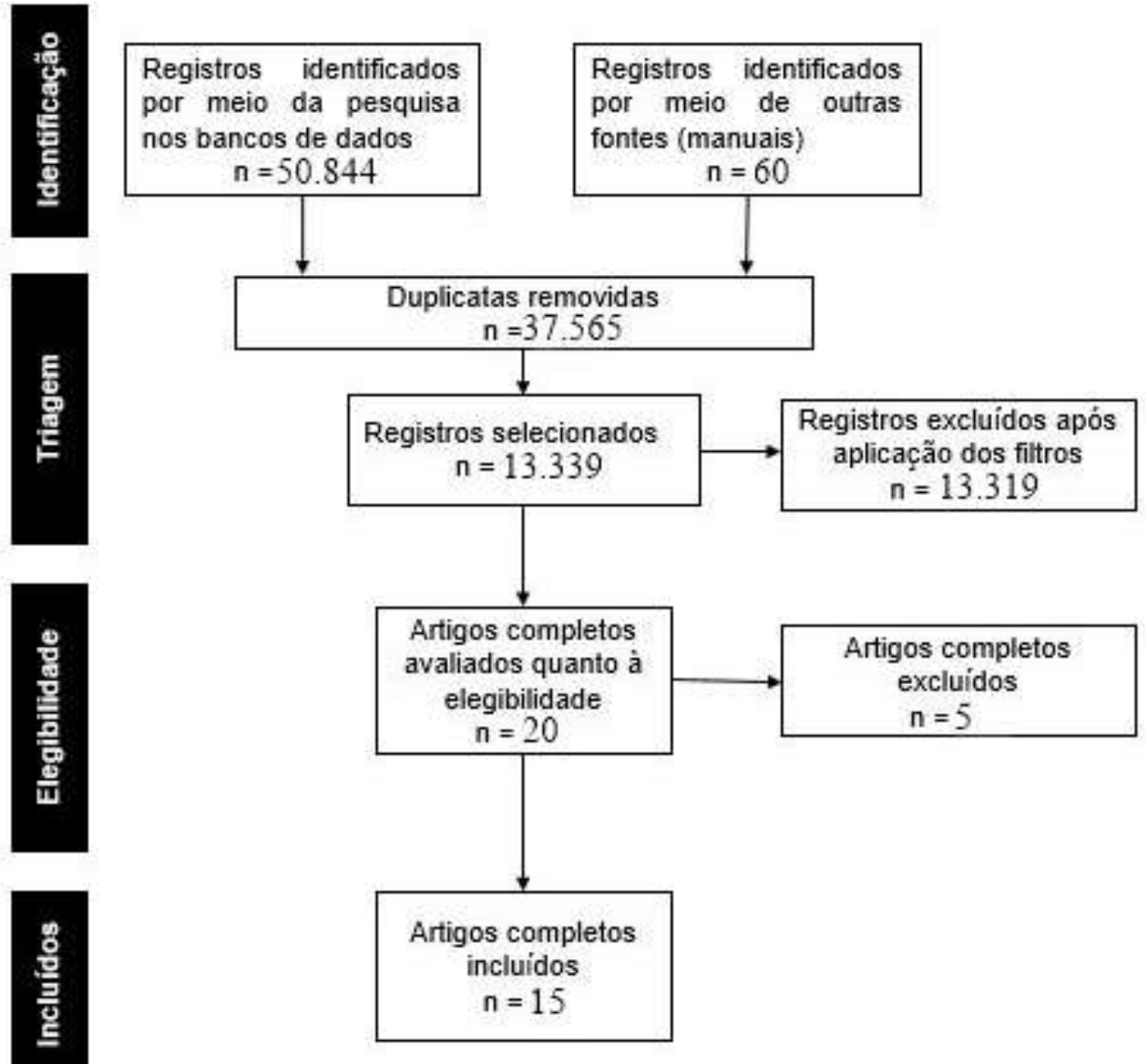

Fonte: Dados de Pesquisa (2021).

Foram realizadas leituras exploratórias para coleta das informações consideradas pertinentes, tendo como base a questão norteadora e o objetivo do estudo. A partir disso, as informações retiradas do texto foram organizadas em um quadro contendo os seguintes dados: autores e ano de publicação; objetivo do estudo; profissionais envolvidos.

\section{Resultados}

A presente pesquisa compreendeu a análise de 15 estudos observacionais, que de alguma forma, avaliaram ações voltadas ao âmbito da saúde mental realizada pelos profissionais da estratégia de saúde da família, possibilitando a comparação das atividades antes e durante a pandemia. Entre esses estudos, 11 foram publicados num contexto antes da pandemia da COVID-19, enquanto que quatro envolveram trabalhos realizados no período da pandemia.

Os profissionais envolvidos foram de Estratégia de Saúde da Família e Centro de Atenção Psicossocial (CAPS). Os estudos selecionados para esta revisão trouxeram as perspectivas dos profissionais, além das limitações observadas para o desenvolvimento das práticas relacionadas à saúde mental. No Quadro 1 encontram-se os artigos selecionados (autores), bem como seus objetivos principais e profissionais envolvidos. 
Quadro 1. Relação dos estudos selecionados para esta revisão integrativa.

\begin{tabular}{|c|c|c|}
\hline Autores (ano) & Objetivo do estudo & Profissionais envolvidos \\
\hline $\begin{array}{l}\text { Arce, Sousa \& } \\
\text { Lima (2011) }\end{array}$ & $\begin{array}{l}\text { Analisar as práticas de Saúde Mental na atuação das equipes da Estratégia } \\
\text { Saúde da Família de Brazilândia, no Distrito Federal, quanto a seus } \\
\text { potenciais e limitações para o cuidado integral aos sujeitos em situações de } \\
\text { sofrimento mental na atenção primária. }\end{array}$ & Equipe de Saúde da Família \\
\hline $\begin{array}{l}\text { Campos et al. } \\
\qquad(2011)\end{array}$ & $\begin{array}{l}\text { Avaliar a articulação entre as redes de atenção primária e de saúde mental } \\
\text { em regiões de alta vulnerabilidade social de uma grande cidade brasileira } \\
\text { (Campinas/SP) através dos parâmetros: pesquisa avaliativa, participativa e } \\
\text { predominantemente qualitativa. }\end{array}$ & Equipe de Saúde da Família \\
\hline $\begin{array}{l}\text { Cavalcante, } \\
\text { Pinto, } \\
\text { Carvalho, Jorge } \\
\text { \& Freitas } \\
\quad(2011)\end{array}$ & $\begin{array}{l}\text { Discutir as práticas de cuidado em saúde mental realizadas pelos } \\
\text { trabalhadores de saúde da Estratégia Saúde da Família no município de } \\
\text { Fortaleza/CE, Brasil. }\end{array}$ & $\begin{array}{l}\text { Médicos, auxiliares de enfermagem, } \\
\text { enfermeiros e agente comunitário de } \\
\text { saúde }\end{array}$ \\
\hline $\begin{array}{l}\text { Sousa et al. } \\
\quad(2011)\end{array}$ & $\begin{array}{l}\text { Analisar o matriciamento como ferramenta articuladora da rede de atenção } \\
\text { em saúde mental. }\end{array}$ & $\begin{array}{l}\text { Profissionais da ESF e Centros de } \\
\text { Atenção Psicossocial }\end{array}$ \\
\hline $\begin{array}{c}\text { Sucigan, } \\
\text { Toledo \& } \\
\text { Garcia }(2012)\end{array}$ & $\begin{array}{l}\text { Compreender como os profissionais de enfermagem de equipes de saúde da } \\
\text { família realizam o acolhimento dos pacientes de saúde mental e seus } \\
\text { sentimentos diante deste trabalho. }\end{array}$ & Auxiliares de enfermagem e enfermeiros \\
\hline $\begin{array}{c}\text { Minoia \& } \\
\text { Minozzo (2015) }\end{array}$ & $\begin{array}{l}\text { Descrever o processo de implantação do acolhimento às pessoas em } \\
\text { sofrimento psíquico em uma unidade de APS, no município de Porto } \\
\text { Alegre/RS. }\end{array}$ & Equipe de saúde da família \\
\hline $\begin{array}{l}\text { Eslabão, } \\
\text { Coimbra, } \\
\text { Kantorski, Pino } \\
\text { \& Santos } \\
\text { (2017) }\end{array}$ & $\begin{array}{l}\text { Analisar a visão de coordenadores da Estratégia Saúde da Família (ESF) } \\
\text { sobre a conformação da rede de saúde mental no município de Pelotas/RS. }\end{array}$ & $\begin{array}{l}\text { Coordenadores da Estratégia Saúde da } \\
\text { Família }\end{array}$ \\
\hline $\begin{array}{l}\text { Gurgel, Jorgel, } \\
\text { Caminha, Maia } \\
\text { Neto \& } \\
\text { Vasconcelos } \\
\text { (2017) }\end{array}$ & $\begin{array}{l}\text { Analisar o cuidado em saúde mental promovido pela equipe de saúde da } \\
\text { família na atenção básica e a prática do apoio matricial. }\end{array}$ & $\begin{array}{l}\text { Médicos, cirurgiões-dentistas, enfermeiros } \\
\text { e familiares de usuários dos serviços }\end{array}$ \\
\hline $\begin{array}{l}\text { Silva et al. } \\
\text { (2019) }\end{array}$ & $\begin{array}{l}\text { Identificar - sob a ótica dos enfermeiros - as potencialidades e limitações } \\
\text { da estratégia do acolhimento direcionada às demandas de saúde mental na } \\
\text { Atenção Básica de um município do Nordeste brasileiro. }\end{array}$ & Enfermeiros \\
\hline $\begin{array}{l}\text { Araújo et al. } \\
\qquad(2020)\end{array}$ & $\begin{array}{l}\text { Apresentar as ações realizadas por duas residentes (psicóloga e enfermeira) } \\
\text { de saúde mental em um Centro de Saúde numa cidade de grande porte no } \\
\text { interior de São Paulo, durante a pandemia da COVID-19. }\end{array}$ & Psicóloga e enfermeira \\
\hline $\begin{array}{l}\text { Barbosa et al. } \\
\qquad(2020)\end{array}$ & $\begin{array}{l}\text { Relatar e analisar a reorganização dos processos de trabalho do CAPS } \\
\text { UERJ, em virtude da política pública que determina o distanciamento } \\
\text { social em meio à pandemia da COVID-19, considerando suas } \\
\text { especificidades e seu compromisso com a lógica territorial. }\end{array}$ & Profissionais da saúde \\
\hline $\begin{array}{l}\text { Cardoso et al. } \\
\qquad(2020)\end{array}$ & $\begin{array}{l}\text { Conhecer o processo de trabalho e o fluxo de atendimento em saúde mental } \\
\text { na Atenção Primária à Saúde sob a ótica dos profissionais da Estratégia } \\
\text { Saúde da Família. }\end{array}$ & $\begin{array}{l}\text { Profissionais da Estratégia Saúde da } \\
\text { Família }\end{array}$ \\
\hline $\begin{array}{l}\text { Carvalho, } \\
\text { Duarte \& } \\
\text { Glanzner } \\
(2020)\end{array}$ & $\begin{array}{l}\text { Avaliar o cuidado em saúde mental à criança no contexto da Estratégia de } \\
\text { Saúde da Família - ESF, a partir da perspectiva dos profissionais. }\end{array}$ & $\begin{array}{l}\text { Profissionais da Estratégia da Saúde da } \\
\text { Família }\end{array}$ \\
\hline
\end{tabular}




\begin{tabular}{|c|l|c|c|}
\hline $\begin{array}{c}\text { Figel et al. } \\
(2020)\end{array}$ & $\begin{array}{l}\text { Apresentar as demandas e ações desenvolvidas com vistas a } \\
\text { instrumentalizar as equipes de saúde que constituem a linha de frente no } \\
\text { atendimento aos doentes e familiares, as quais também apresentam } \\
\text { sofrimento mental neste momento único da saúde pública mundial. }\end{array}$ & Equipe multiprofissional \\
\hline $\begin{array}{c}\text { Nabuco, } \\
\text { Oliveira \& } \\
\text { Afonso (2020) }\end{array}$ & $\begin{array}{l}\text { Apresentar uma proposta para a atuação das equipes de Atenção Primária } \\
\text { no enfrentamento ao adoecimento mental relacionado à pandemia. }\end{array}$ & Equipe multiprofissional \\
\hline
\end{tabular}

Fonte: Dados coletados dos 15 artigos selecionados para esta revisão.

No Quadro 2 têm-se especificadas as estratégias de atuação da ESF no contexto da atenção à saúde mental, tanto antes quanto no período da pandemia. Entre as ações utilizadas, o apoio matricial, visita domiciliar, ação do agente comunitário de saúde, identificação das famílias com fatores de risco para o adoecimento mental relacionado à pandemia e orientações à população para minimizar o adoecimento mental durante o confinamento caracterizam-se como pontos fundamentais da atividade da APS neste contexto pandêmico, refletindo adaptações do serviço à nova realidade e demanda.

Quadro 2. Relação das estratégias abordadas pelos profissionais de saúde no contexto da saúde mental no serviço da atenção básica.

\begin{tabular}{|c|c|}
\hline Autores (ano) & Principais estratégias \\
\hline Sousa et al. (2011) & Apoio matricial. \\
\hline Sucigan, Toledo \& Garcia (2012) & Tecnologias relacionais. \\
\hline Araújo et al. (2020) & Visita domiciliar. \\
\hline $\begin{array}{l}\text { Figel, Sousa, Yamaguchi, Gonçalo, } \\
\text { Murta \& Alves (2020) }\end{array}$ & Terapia comunitária. \\
\hline Cardoso et al. (2020) & Apoio do agente comunitário de saúde. \\
\hline Figel et al. (2020) & Reuniões de equipes. \\
\hline Moreira et al. (2020) & Teleatendimento e videochamadas. \\
\hline Nabuco, Oliveira \& Afonso (2020) & $\begin{array}{l}\text { Implementação de grupos de trabalhos compostos por membros da gestão e trabalhadores, } \\
\text { objetivando realizar discussões e deliberar ações voltadas à manutenção da produção de } \\
\text { cuidados e ao aprimoramento da prevenção dos usuários e colaboradores. }\end{array}$ \\
\hline Moreira et al. (2020) & $\begin{array}{l}\text { Articulação do cuidado territorial, intra e intersetorial, e acompanhamento intensivo, por meio } \\
\text { telefônico. }\end{array}$ \\
\hline $\begin{array}{l}\text { Figel, Sousa, Yamaguchi, Gonçalo, } \\
\text { Murta \& Alves (2020) }\end{array}$ & $\begin{array}{l}\text { Identificação das famílias como fatores de risco para adoecimento mental relacionado à } \\
\text { pandemia (infecção pelo COVID-19; transtorno mental pré-existente; idade avançada; } \\
\text { vulnerabilidade social). }\end{array}$ \\
\hline $\begin{array}{l}\text { Gurgel, Jorge, Caminha, Maia Neto \& } \\
\text { Vasconcelos (2017) }\end{array}$ & $\begin{array}{l}\text { Articulação intersetorial (educação, saúde, cultura, organizações comunitárias) para viabilizar } \\
\text { respaldo às demandas das famílias em maior vulnerabilidade (segurança, alimentação e abrigo). }\end{array}$ \\
\hline $\begin{array}{l}\text { Gurgel, Jorge, Caminha, Maia Neto \& } \\
\text { Vasconcelos (2017) }\end{array}$ & $\begin{array}{l}\text { Orientações à população que minimizem o adoecimento mental durante o confinamento: limitar } \\
\text { exposição às notícias relacionadas à COVID-19 que causam ansiedade, procurar fontes } \\
\text { confiáveis e oficiais de informação, interagir com familiares e amigos à distância e manter rotina } \\
\text { saudável. }\end{array}$ \\
\hline $\begin{array}{l}\text { Gurgel, Jorge, Caminha, Maia Neto \& } \\
\text { Vasconcelos (2017) }\end{array}$ & Apoio para minimizar as barreiras para vivência do luto daqueles que perderam entes queridos. \\
\hline
\end{tabular}

Fonte: Dados coletados dos 15 artigos selecionados para esta revisão.

Muitos estudos também trouxeram as limitações de acordo com cada abordagem (Quadro 3). Segundo as limitações apresentadas pelos profissionais de saúde no contexto da saúde mental na atenção básica, verifica-se a fragilidade do sistema 
quanto à oferta de serviços efetivos aos pacientes com transtornos mentais, ressaltando-se uma rede de saúde mental fragmentada, com profissionais sem habilidades na área, sobrecarga de trabalho e espaço físico insuficiente. Estes aspectos reforçam a necessidade de desenvolvimento de políticas públicas e investimentos voltados ao serviço de saúde mental na atenção básica, visando a criação de estratégias que contemplem integralmente a necessidade dos usuários. A baixa adesão ao serviço pode ser consequência dos déficits no processo de assistência, comprometendo a frequência do paciente à unidade e, por conseguinte, o tratamento, acentuando as complicações relacionadas ao respectivo transtorno.

Quadro 3. Relação das limitações apresentadas pelos profissionais de saúde encontradas no contexto da saúde mental na atenção básica.

\begin{tabular}{|c|c|}
\hline Autores (ano) & Principais limitações \\
\hline $\begin{array}{l}\text { Moraes, Araújo, Porto, Trajano \& } \\
\text { Sousa (2021) }\end{array}$ & Dificuldade de acesso ao cuidado em saúde mental, por não haver rede de apoio estruturada. \\
\hline $\begin{array}{l}\text { Silva et al. (2019); } \\
\text { Estebão, Coimbra, Kantorski, Pino } \\
\quad \& \text { Santos (2017) }\end{array}$ & $\begin{array}{l}\text { Baixa capacidade de resolutividade no âmbito da saúde da família, uma vez que as ações privilegiam } \\
\text { consultas ambulatoriais médicas e encaminhamentos para internações. }\end{array}$ \\
\hline Araújo et al. (2020) & Práticas de promoção de saúde pouco consolidadas. \\
\hline $\begin{array}{l}\text { Estebão, Coimbra, Kantorski, Pino } \\
\qquad \text { \& Santos (2017) }\end{array}$ & $\begin{array}{l}\text { Deficiência na formação/ capacitação pela maioria dos profissionais da atenção básica, devido a uma } \\
\text { perdura do modelo patológico/ curativo de atenção em saúde. }\end{array}$ \\
\hline $\begin{array}{l}\text { Cavalcante, Pinto, Carvalho, Jorge } \\
\qquad \& \text { Freitas (2011) }\end{array}$ & Redes de saúde mental ocorrem de forma frágil, sem diálogo necessário. \\
\hline Arce, Sousa \& Lima (2011) & $\begin{array}{l}\text { Ausência de profissional/ pessoal preparado para realizar o cuidado visando a integralidade do sujeito } \\
\text { e de seus familiares, e também o insight necessário para internar nas situações que se apresentam no } \\
\text { cotidiano da assistência na atenção primária à saúde. }\end{array}$ \\
\hline $\begin{array}{l}\text { Moraes, Araújo, Porto, Trajano \& } \\
\text { Sousa (2021) }\end{array}$ & Não ter contrarreferência. \\
\hline Sousa et al. (2011) & Espaço físico insuficiente. \\
\hline Araújo et al. (2020) & Sobrecarga de trabalho para apenas um profissional. \\
\hline Moreira et al. (2020) & Usuários não dispõem de conexão de internet satisfatória, ou de telefone. \\
\hline $\begin{array}{l}\text { Cavalcante, Pinto, Carvalho, Jorge } \\
\text { \& Freitas (2011) }\end{array}$ & Baixa adesão. \\
\hline Arce, Sousa \& Lima (2011) & Questões culturais. \\
\hline
\end{tabular}

Fonte: Dados coletados dos 15 artigos selecionados para esta revisão.

\section{Discussão}

A pandemia da COVID-19 culminou na necessidade de reorganização da atenção à saúde, incluindo a saúde mental, intimamente relacionada ao impacto no cotidiano de vida e saúde (Figel, Sousa, Yamaguchi, Gonçalo, Murta \& Alves, 2020). Sabe-se que em situações de surtos ocorre amplo e variável espectro de manifestações de adoecimento mental. Precipitam-se transtornos mentais em pessoas sem doença mental, agravam-se aqueles com doença mental pré-existente e tornam-se mais susceptíveis os familiares de infectados (Araújo et al., 2020).

Mais recentemente, em revisão sistemática e meta-análise realizada por Wu et al. (2021), foi observada uma prevalência geral combinada de depressão, ansiedade, angústia e insônia de 31,4\%, 31,9\%, 41,1\% e 37,9\%, respectivamente. Além disso, os pacientes com doenças crônicas não infecciosas, pessoas em quarentena e pacientes com COVID-19 apresentaram maior risco de depressão $(\mathrm{Q}=26,73, \mathrm{p}<0,01)$ e ansiedade $(\mathrm{Q}=21,86, \mathrm{p}<0,01)$ do que outras populações (Wu et al., 2021). 
Tendo em vista todos os problemas relacionados à saúde mental, a atenção primária a saúde, como porta de entrada aos serviços, torna-se de grande importância aos usuários principalmente no período de pandemia. Nesta revisão foram incluídos trabalhos que apresentaram estratégias e limitações antes da pandemia e outros artigos com estratégias para o período da pandemia. O objetivo dessas inclusões foi o fato de ser necessário conhecer os problemas pré-existentes e a necessidade de adaptar-se ao novo. Além disso, observou-se uma escassez de trabalhos com orientações e limitações referentes ao período da pandemia.

O potencial dessa esfera de atenção pode ser diminuído pelo seu insucesso, principalmente pelo despreparo, sobrecarga ou desinteresse dos profissionais envolvidos, a falta desses profissionais o que resulta em equipes incompletas, dificuldades de garantir a integralidade do cuidado (referência e contrarreferência) e a escassez de recursos disponibilizados (Moraes, Araújo, Porto, Trajano \& Sousa, 2021).

Contudo, Silva et al. (2019) relataram que é possível visualizar algumas fragilidades na operacionalização do acolhimento na Atenção Básica. Uma delas diz respeito a compreensão do acolhimento como um espaço físico, que deve ser realizado somente na chegada dos(as) usuários e somente pelos profissionais da saúde, confundindo-o com a triagem e diminuindo a sua complexidade (Sousa et al., 2011). Além disso, em seu estudo, os autores afirmaram que, mediatizado por tecnologias leves como a humanização, empatia, escuta ativa dos usuários e a corresponsabilização do cuidado, o acolhimento se torna potente e viável (Silva et al., 2019).

Entretanto, há limitações neste processo por despreparo dos(as) profissionais, aos problemas sociais da comunidade, aos vínculos debilitados da família e a fragilidade da Rede de Atenção Psicossocial (Arce, Sousa \& Lima, 2011). Ademais, os autores ainda concluíram que, como estratégias de enfrentamento sugere-se a interlocução entre os serviços da rede de saúde mental através de um fluxograma municipal; a educação permanente dos(as) profissionais atuantes nos serviços; e o apoio qualificado do Núcleo Ampliado da Saúde da Família junto às Unidades Básicas de Saúde.

No estudo de Gurgel, Jorge, Caminha, Maia Neto e Vasconcelos (2017), por meio de aplicação de entrevistas semiestruturadas com 14 profissionais de saúde e sete familiares de usuários de unidades de saúde da família, os autores concluíram que o apoio matricial é uma estratégia inovadora para expandir conhecimentos acerca do cuidado em saúde mental e contribuir para que haja capacidade resolutiva na Atenção Básica, evidenciando a importância de uma responsabilização dos profissionais da atenção básica para com o cuidado em saúde mental, por meio de uma capacitação que lhes permita compreender e colocar em prática ações que vão além de prescrições. "É papel da APS o trato e o cuidado de pacientes em saúde mental, antes, durante e após o encaminhamento, se este for realizado" (Moraes, Araújo, Porto, Trajano \& Sousa, 2021, p. 10486).

Além disso, os autores enfatizaram que o acesso dos usuários através do apoio matricial, segundo o olhar de seus familiares, tem sido visto como algo positivo, pela facilitação que é gerada através da existência de uma unidade de saúde próxima às suas residências, porém os familiares têm sentido a falta de resolução dos problemas, além da sobrecarga física e emocional que o cuidado lhes proporciona (Estebão, Coimbra, Kantorski, Pino \& Santos, 2017). O apoio matricial em saúde objetiva assegurar retaguarda especializada a equipes e profissionais encarregados da atenção a problemas de saúde. Trata-se de uma metodologia de trabalho complementar àquela prevista em sistemas hierarquizados, a saber: mecanismos de referência e contrarreferência, protocolos e centros de regulação (Campos et al., 2011).

É fundamental o desenvolvimento de educação permanente e em serviço dos profissionais, especialmente quanto ao acolhimento, manejo clínico e comunitário dos sujeitos em sofrimento psíquico, bem como a efetivação do matriciamento do cuidado.

Por conseguinte, dentre os trabalhos selecionados no período da pandemia da COVID-19, Figel e colaboradores (2020) apresentaram e discutiram as demandas e ações desenvolvidas com vistas a instrumentalizar as equipes de saúde que 
constituem a linha de frente no atendimento aos doentes e familiares, as quais também apresentam sofrimento mental neste momento único da saúde pública. Neste relato de experiência, os autores trouxeram uma nova forma de acolhimento psicológico, por meio de acesso telefônico, visando oferecer apoio, inclusive direcionado aos profissionais de saúde. Essa modalidade de acolhimento contribui para um melhor entendimento dos sintomas pelos próprios pacientes, a fim de que haja uma procura pelo atendimento de forma mais direcionada.

Apesar de os recursos tecnológicos terem um impacto positivo durante a pandemia, Carvalho, Duarte \& Glanzner (2020), mostraram algumas desvantagens desses recursos que dizem respeito às dificuldades de acesso de usuários que não dispõem de conexão de internet satisfatória, ou de telefone. Além disso, alguns usuários têm baixa adesão a essas modalidades, muitas vezes por apresentarem dificuldades no uso desses equipamentos, ou em virtude da ausência de privacidade necessária na residência, que não permite falar abertamente sobre questões sigilosas ou de foro íntimo. Segundo Cardoso e colaboradores (2020), questões culturais, como viver em área rural e/ ou não valorizar o uso de dispositivos tecnológicos, também contribuem para que esses usuários apresentem mais resistência a utilizar a tecnologia para atendimento de saúde, principalmente entre idosos.

Mesmo no contexto da pandemia, no trabalho realizado por Minoia e Minozo (2015), é destacada a importância dos agentes comunitários de saúde na identificação dos grupos com fatores de risco à doença mental. Ademais, observa-se ainda a necessidade da presença desses profissionais para um acolhimento mais próximo dos usuários, sendo estes profissionais que de certa forma mais conhecem o perfil de cada família.

Em scoping review realizada por Moreira e colaboradores (2020), com objetivo de mapear as evidências sobre intervenções em saúde mental implementadas durante a pandemia da COVID-19 no mundo, evidenciaram-se as práticas de telemonitoramento, como visto nesta revisão com trabalhos realizados na atenção primária. Além disso, os autores Sucigan, Toledo \& Garcia (2012), encontram outras práticas como jogos virtuais e estratégias focalizadas no suporte social e em técnicas de relaxamento muscular, caracterizadas como não farmacológicas e de baixo custo.

Apesar da escassez de estudos sobre as práticas de saúde mental na atenção primária à saúde durante a pandemia da COVID-19, observa-se que a utilização dos recursos tecnológicos para o acolhimento foi uma alternativa interessante e eficaz para o monitoramento e acompanhamento dos usuários (Barbosa et al., 2020). No entanto, vale ressaltar a existência de usuários que não têm acesso às tecnologias e/ou são resistentes a estas. Além disso, destaca-se a necessidade da implementação de ações que visem o esclarecimento à população em geral quanto a importância das novas práticas de acompanhamento neste período, além de informá-los continuamente sobre as medidas de prevenção à COVID-19.

\section{Considerações Finais}

No cenário atual da pandemia da COVID-19 é notório o aumento de transtornos mentais na população em geral, assim como agravamento dos casos pré-existentes. Dessa forma, o acolhimento em saúde mental na atenção primária à saúde é indispensável para esses usuários. Apesar da escassez de estudos na literatura, pode-se concluir que há uma implementação de novas práticas adaptadas ao novo cenário, como por exemplo o teleatendimento, por meio de videochamadas e ligações.

No entanto, essas práticas possuem limitações em decorrência do perfil dos usuários. Dessa forma, são necessárias novas estratégias para acolhimento que possam incluir todos os usuários do serviço. Torna-se crucial o incentivo por parte do governo federal direcionado ao aprimoramento dessas práticas em saúde mental na esfera da atenção básica, considerando-se a crescente demanda associada à evolução da pandemia no país.

Além disso, diante das informações supracitadas observa-se uma evidente necessidade de estudos voltados ao tema. São essenciais pesquisas que abordem melhores estratégias de acolhimento para pacientes com transtornos mentais a nível de Atenção Básica, e assim, possa contribuir para reduzir as demandas do serviço especializado, "desafogando-o", bem como a 
apresentação de formas mais eficazes de interligação da Rede de Apoio a esses pacientes, de modo a garantir a integralidade do atendimento.

\section{Referências}

Alves, G. G. \& Aerts, D. (2011). As práticas educativas em saúde e a Estratégia Saúde da Família. Ciência e Saúde Coletiva, 16(1), 319-325.

Araújo, B. P. M., Pacciulio, A. L. M., Montanha, L. T., Emerich, B. F., Pellati, G. \& Campos, R. O. (2020). Pandemia de COVID-19 e a implementação de teleatendimentos em saúde mental: um relato de experiência na Atenção Básica. Saúde em Redes, 6(2), 7-13.

Arce, V. A. R., Sousa, M. F. \& Lima, M. G. (2011). A práxis da Saúde Mental no âmbito da Estratégia Saúde da Família: contribuições para a construção de um cuidado integrado. Physis Revista de Saúde Coletiva, 2(2) 541-560.

Arruda, D. É. G., Martins, D. D. S., Silva, I. F. M., \& Sousa, M. N. A. (2020). Prognóstico de pacientes com COVID-19 e doenças crônicas: uma revisão sistemática. Comunicação em Ciências da Saúde, 31(3), 79-88.

Baloch, S., Baloch, M. A., Zheng, T. \& Pei, X. (2020). The Coronavirus Disease (COVID-19) Pandemic. Tohoku J Exp Med, $250,271-280$.

Barbosa, A. S., Nascimento, C. V., Dias, L. B. S., Santo, T. B. E., Chaves, R. C. S. \& Fernandes, T. C. (2020). Processo de trabalho e cuidado em saúde mental no Centro de Atenção Psicossocial da UERJ na pandemia de COVID-19. Brazilian Journal of Health and Biomedical Sciences, 19 (1), 11-19.

Baxter, A. J., Patton, G., Scott, K. M., Degenhardt, L. \& Whiteford, A. (2013). Global epidemiology of mental disorders: what are we missing? Plos One, 8(6), 65514.

Bezerra, A. L. D., Rodrigues, E. J. F., Souto, L. H. G. G., Nóbrega, A. R. O., Silva, N. S., \& Sousa, M. N. A. (2020). Atuação de uma equipe multiprofissional em tempos de coronavírus. Revista Interdisciplinar em Saúde, 7(1), 1993-2008.

Brasil. Ministério da Saúde (2021). Brasil confirma primeiro caso da doença. https://www.saude.gov.br/noticias/agencia-saude/46435-brasil-confirmaprimeiro-caso-de-novo-coronavirus.

Brasil. Ministério da saúde. Secretaria de Atenção à Saúde (2005). Reforma Psiquiátrica e Política de Saúde Mental no Brasil. http://portal.saude.gov.br/portal/arquivos/pdf/relatorio_15_anos_caracas.pdf.

Brasil. Ministério da Saúde. Secretaria de Atenção à Saúde (2006). Departamento de Atenção Básica. Política Nacional de Atenção Básica. http://bvsms.saude.gov.br/bvs/publicacoes/politica_nacional_atencao_basica_2006.pdf.

Campos, G. W. S. \& Domitti, A. C. (2007). Apoio matricial e equipe de referência: uma metodologia para gestão do trabalho interdisciplinar em saúde. Cadernos de Saúde Pública, 23(2), 399-407.

Campos, R. O., Gama, A. C., Ferrer, A. L., Santos, D. V. D., Stefanello, S. Trapé, L. T. \& Porto, K. (2011). Saúde mental na atenção primária à saúde: estudo avaliativo em uma grande cidade brasileira. Ciência e Saúde Coletiva, 16(12), 4643-4652.

Cardoso, L. C. B., Pinho, L. B. D., Arruda, G. O. D., Marcon, S. S., Giacon-Arruda, B. C. C., \& Paiano, M. (2020). Processo de trabalho e fluxo de atendimento em saúde mental na atenção primária à saúde. Texto e Contexto Enfermagem, 29, 20190191.

Carvalho, J., Duarte, M. L. C., \& Glanzner, C. H. (2020). Child mental health care in the context of the Family Health Strategy: an evaluative study. Revista Gaúcha de Enfermagem, 41, 20190113.

Cavalcante, C. M., Pinto, D. M., Carvalho, A. Z. T., Jorge, M. S. B. \& Freitas, C. H. A. (2011). Desafios do cuidado em saúde mental na estratégia saúde da família. Revista Brasileira em Promoção da Saúde, 24(2), 102-108.

Coimbra, V. C. C., Oliveira, M. M., Vila, T. C. \& Almeida, M. C. P. (2005). A atenção em saúde mental na estratégia saúde da família. Revista Eletrônica de Enfermagem, 7(1), 111-113.

Correia, V. R., Barros, S. \& Colvero, L. A. (2011). Saúde mental na atenção básica: prática da equipe de saúde da família. Revista da Escola de Enfermagem da USP, 45(6), 1501-1506.

Duarte, M. Q., Santos, M. A. S., Lima, C. P., Giordani, J. P. \& Trentini, C. M. (2020). Covid-19 and the impacts on mental health: a sample from Rio Grande do Sul, Brazil. Ciência de Saúde Coletiva, 25(9), 3401-3411.

Eslabão, A. D., Coimbra, V. C. C., Kantorski, L. B., Pino, L. B. \& Santos, E. O. (2017). Mental health care network: the views of coordinators of the Family Health Strategy (FHS). Revista Gaúcha de Enfermagem, 38(1), 210-219.

Figel, F. C., Sousa, M. C., Yamaguchi, L. S., Gonçalo, S. L., Murta, J. E. \& Alves, A. C. (2020). Reorganização da atenção à saúde mental na pandemia de Covid-19. Revista de Saúde Pública do Paraná, 3(1), 118-128.

Gazignato, E. C. S. (2014a). Saúde mental na atenção básica: o trabalho em rede e o matriciamento em saúde mental na Estratégia de Saúde da Família. Saúde em Debate, 38(101), 296-304.

Gurgel, A. L. L. G., Jorgel, M. S. B., Caminha, E. C. C., Neto, J. P. M. \& Vasconcelos, M. G. F. (2017). Cuidado em saúde mental na estratégia saúde da família: a experiência do apoio matricial. Revista de Enfermagem UERJ, 25, e7101.

Hossain, M. M., Sultana, A. \& Purohit, N. (2020). Mental health outcomes of quarantine and isolation for infection prevention: a systematic umbrella review of the global evidence. Epidemiology and Health, 42, e2020038. 
L' Abbate, S. (2003). A análise institucional e a saúde coletiva. Revista Ciência e Saúde Coletiva, 8(1), $134-142$.

Minoia, N. P. \& Minozzo, F. (2015). Acolhimento em saúde mental: operando mudanças na Atenção Primária à Saúde. Psicologia: Ciência e Profissão, 35(4), 1340-1349.

Moraes, L. G. A., Araujo, R. M. S., Porto, R. M., Trajano, J. A. \& Sousa, M. N. A. (2021). Saúde mental: o papel da atenção primária à saúde. Brazilian Journal of Health Review, 4(3), 1475-1489.

Moreira, W. C., Sousa, K. H. J. F., Sousa, A. R., Santana, T. S. Zeitoune, R. C. G. \& Nobrega, M. P. S. S. (2020). Mental health interventions in times of COVID-19: a scoping review. Scielo Preprints. https://doi.org/10.1590/SciELOPreprints.1007.

Mowbray, P. K., Wilkinson, A., \& Tse, H. H. (2014). An integrative review of employee voice: Identifying a common conceptualization and research agenda. International Journal of Management Reviews, 17(3), 382-400.

Nabuco, G., Oliveira, M. H. P. P. \& Afonso, M. P. D. (2020). O impacto da pandemia pela COVID-19 na saúde mental: qual é o papel da Atenção Primária à Saúde? Revista Brasileira de Medicina Família e Comunidade, 15(42), 2532-2541.

Ribeiro, M. O. P., Caccia-Bava, M. C. G. \& Guanaes-Lorenzi, C. (2013). Atenção à saúde mental na Estratégia Saúde da Família: recursos não reconhecidos. Psicologia USP, 24(3), 369-390.

Rodrigues, J. \& Brognoli, F. (2014). Acolhimento no serviço de atenção psicossocial. Cadernos Brasileiros de Saúde Mental, 6(13), 61-74.

Santos, E. G. \& Siqueira, M. M. (2010). Prevalência dos transtornos mentais na população adulta brasileira: uma revisão sistemática de 1997 a 2009. Jornal Brasileiro de Psiquiatria, 59(3), 238-246.

Scoz, T. M. X. \& Fenili, R. M. (2003). Como desenvolver projetos de atenção à saúde mental no programa de saúde da família. Revista Eletrônica de Enfermagem, 5(2), 71-77.

Silva, P. M. C., Costa, N. F., Barros, D. R. R. E., Silva, J. A., Silva, J. R. L., \& Brito, T. S. (2019). Saúde mental na atenção básica: possibilidades e fragilidades do acolhimento. Revista Cuidarte, 10(1), e617.

Sousa, F. S. P. D., Jorge, M. S. B., Vasconcelos, M. G. F., Barros, M. M. M. A. D., Quinderé, P. H. D., \& Gondim, L. G. F. (2011). Tecendo a rede assistencial em saúde mental com a ferramenta matricial. Physis: Revista de Saúde Coletiva, 21(4), 1022-1034.

Sousa, M. N. A., Estrela, Y. D. C. A., \& Bezerra, A. L. D. (2020). Perfil epidemiológico de casos de coronavírus no estado da Paraíba utilizando o Boletim Epidemiológico Local. Inf. Pauta, 5(2), 92-106.

Souza, L. G. S. (2012). Saúde Mental na Estratégia Saúde da Família: revisão da literatura brasileira. Revista Saúde e Sociedade, 21 (4), 1022-1034.

Souza, R. C. (2004). Produção de sentidos por profissionais de saúde da família acerca do doente mental e dos cuidados a ele dirigidos (Tese de Doutorado). Programa de Pós-Graduação em Enfermagem, Escola de Enfermagem de Ribeirão Preto, Universidade de São Paulo, Ribeirão Preto.

Sucigan, D. H. I., Toledo, V. P. \& Garcia, A. P. R. F. (2012). Acolhimento em saúde mental: desafio profissional na estratégia saúde da família. Revista Rene, $13(1), 2-10$

World Healt Organization. (2013). Investing in mental health: evidence for action.

Wu, T., Jia, X., Shi, H., Niu, J., Yin, X., Xie, J. \& Wang, X. (2021). Prevalence of mental health problems during the COVID-19 pandemic: A systematic review and meta-analysis. Science Direct, 281, 91-98. 